\title{
Potential of Hydrophilic Interaction Chromatography for the Analytical Characterization of Protein Biopharmaceuticals
}

\author{
Aurélie PERIAT ${ }^{(1)}$, Szabolcs FEKETE(1), Alessandra CUSUMANO(1), Jean-Luc VEUTHEY ${ }^{(1)}$, \\ Alain BECK ${ }^{(2)}$, Matthew LAUBER ${ }^{(3)}$, Davy GUILLARME ${ }^{(1)}$
}

(1) School of Pharmaceutical Sciences, University of Geneva, University of Lausanne, Boulevard d'Yvoy 20, 1211 Geneva 4, Switzerland

(2) Center of Immunology Pierre Fabre, 5 Avenue Napoléon III, BP 60497, Saint-Julienen-Genevois 74160, France

(3) Waters Corporation, 34 Maple Street, Milford, Massachusetts 01757-3696, United States

CORRESPONDENCE: davy.guillarme@unige.ch

\begin{abstract}
A new stationary phase based on wide-pore hybrid silica bonded with amide ligand has been used to explore the utility of HILIC for the analytical characterization of protein biopharmaceuticals. Various, highly-relevant samples were tested, including different insulins, interferon $a-2 b$ and trastuzumab. This work shows that HILIC can be successfully employed for the analysis of therapeutic proteins and $m A b s$, using mobile phase compositions comprised of between 65 and $80 \%$ ACN and $0.1 \%$ TFA. In terms of elution order and selectivity, these HILIC separations have proven to be highly orthogonal to RPLC, while the kinetic performance remains comparable. In the case of characterizing trastuzumab, HILIC was uniquely able to resolve several important glycoforms at the middleup level of analysis (fragments of $25-100 \mathrm{kDa}$ ). Such a separation of glycoforms has been elusive by other separation mechanisms, such as RPLC and IEX. Besides showing orthogonality to RPLC and improved separations of glycoforms, HILIC offers several additional benefits for biopharmaceutical characterization: i) an inherent compatibility with MS, ii) a reduced requirement for very high mobile phase temperatures that are otherwise needed in RPLC to limit undesirably strong adsorption to the surface of the stationary phase, and iii) the possibility to couple several columns in series to improve resolving power, thanks to comparatively low mobile phase viscosity.
\end{abstract}

\section{Keywords}

Hydrophilic interaction chromatography; HILIC; Insulins; interferon a-2b; trastuzumab 


\section{Introduction}

The importance of biotechnology products has been ever increasing, and it is expected that the majority of new drug approvals will be biologics [1,2]. Therapeutic proteins are more complex than small molecules due to their high molecular weights, numerous possible conformations, post-translational modifications and microheterogeneity [3]. The heterogeneity of biopharmaceuticals is a consequence of their expression from living organisms and the fact that modifications can readily occur during production, extraction, purification, formulation and storage [4]. These changes can affect the efficacy and safety of biopharmaceuticals. Therefore, it is important that they be properly characterized. For this purpose, a variety of orthogonal analytical techniques can be used, including liquid chromatography, gel and capillary electrophoresis, mass spectrometry (MS), and spectroscopy [5].

Liquid chromatography is an indispensable tool for intact protein analysis and different modes can be employed, such as reversed-phase liquid chromatography (RPLC), sizeexclusion chromatography (SEC), ion-exchange chromatography (IEX), hydrophobic interaction chromatography (HIC) and hydrophilic interaction chromatography (HILIC) $[6,7]$. SEC, IEX and HIC are historical techniques, well established in the biopharmaceutical industry for intact proteins analysis, though they suffer from a difficult hyphenation to MS. For this reason, RPLC in combination with UV, fluorescence and, above all, MS is more and more widely used today to assess protein batch purity and highlight any protein degradation or misfolds [8-10]. In the RPLC of large proteins, an analyst often encounters adsorption, carryover, multiple peak formation and low chromatographic performance due to slow diffusion and secondary interactions with stationary phase. Nonetheless, it has been shown that RPLC of large proteins can be enhanced through the use of elevated mobile phase temperature (in the range 60 to $90^{\circ} \mathrm{C}$ ), addition of ion-pairing agents to the mobile phase (e.g. TFA), use of columns packed with wide-pore particles of $300-1000 \AA$ pore sizes, and/or use of silica-based stationary phases with reduced silanol amount and/or acidity [2].

HILIC is a variant of normal phase chromatography in which the stationary phase is polar and the mobile phase consists of water and 60 to $95 \%$ of an aprotic, miscible organic solvent, usually acetonitrile (ACN). The highly organic mobile phase is particularly well suited to ESI-MS detection, leading to substantial improvements in sensitivity for a large variety of compounds [11,12]. The high organic content of the mobile phase also generates relatively low back pressures, allowing the use of high flow rates or long columns packed with small particles. The retention mechanism of HILIC is based mainly on hydrophilic partitioning of polar compounds between a water-enriched layer formed at the surface of the stationary phase and the highly organic mobile phase. Depending on the nature of both the stationary 
phase and the mobile phases, as well as the physico-chemical properties of the analytes, additional interaction mechanisms can occur, including hydrogen bonding, dipole-dipole interactions, and ionic interactions [13-15]. HILIC is nowadays largely employed for the analysis of polar and/or ionizable small compounds, peptides, and glycans [16-18]. However, it has not yet been applied for the analysis of intact proteins, except for the characterization of lipophilic membrane proteins, which could bind irreversibly to a stationary phase under RPLC conditions [18-21].

In this first study of its kind, a new wide-pore, amide-bonded stationary phase has been used to investigate the applicability of HILIC to the characterization of intact protein biopharmaceuticals. Compelling separations have been obtained for a set of highly relevant biopharmaceuticals, including insulins, interferon $a-2 b$, and trastuzumab. Interestingly, it has been found that the performance of these novel HILIC separations nicely complements the capabilities of conventional separation mechanisms, such as RPLC and IEX. 


\section{Experimental part}

\subsection{Reagents and analytes}

Water was obtained from a Milli-Q Water Purification System from Millipore (Bedford, MA, USA). Acetonitrile (ACN) and methanol $(\mathrm{MeOH})$ were ULC-MS grade and purchased from Biosolve (Valkenswaald, Netherlands). Trifluoroacetic acid (TFA) was obtained from SigmaAldrich (Buchs, Switzerland). Tris(hydroxymethyl)aminomethane (Tris) and $1 \mathrm{M}$ sodium hydroxide $(\mathrm{NaOH})$ solution were purchased from Sigma-Aldrich (Buchs, Switzerland).

Human insulin (Insuman rapid $100 \mathrm{UI} / \mathrm{mL}$ ), insulin glargine (Lantus $100 \mathrm{UI} / \mathrm{mL}$ ) and insulin glulisine (Apidra $100 \mathrm{UI} / \mathrm{mL}$ ) were obtained from Sanofi Aventis (Paris, France). Insulin lispro (Humalog $100 \mathrm{UI}$ ) and insulin aspart (Novorapid $100 \mathrm{UI} / \mathrm{mL}$ ) were purchased from Eli Lilly (Indianapolis, USA) and Novo Nordisk Pharma AG (Küsnacht, Switzerland), respectively. Bovine insulin was purchased from Sigma-Aldrich (Buchs, Switzerland). The sequences of human, bovine and synthetic insulins are provided in Table 1.

Dithiothreitol (DTT), hydrogen peroxide $30 \%$ and methionine were purchased from SigmaAldrich. IdeS enzyme (FabRICATOR ${ }^{\mathrm{TM}}$ ) and EndoS2 enzyme (GlycINATOR ${ }^{\mathrm{TM}}$ ) were purchased from Genovis Inc. (Lund, Sweden). Recombinant interferon $a-2 b$ was purchased from Merck (Darmstadt, Germany). Trastuzumab was kindly provided by Pierre Fabre laboratories (Saint-Julien en Genevois, France).

\subsection{Instrumentation and columns}

RPLC and HILIC analyses of insulins were performed on a Waters ACQUITY UPLC H-Class system (Milford, MA, USA). This system included a quaternary solvent delivery pump, an autosampler, and a column oven. The autosampler included a flow through needle (FTN) injection system with a $15 \mu \mathrm{L}$ needle. The system was equipped with a UV-DAD detector set at $214 \mathrm{~nm}$. The dwell volume $\left(\mathrm{V}_{\mathrm{d}}\right)$ was experimentally measured as $375 \mu \mathrm{L}$. Data acquisition, data handling and instrument control were performed with Empower 3 (Waters).

The RPLC and HILIC analyses of interferon $a-2 b$ and trastuzumab samples were performed on a Waters ACQUITY UPLC I-Class system equipped with a binary solvent delivery pump, an autosampler, a UV-DAD and a fluorescence detector (FD) set at $\lambda_{\mathrm{ex}}=280 \mathrm{~nm}$ and $\lambda_{\mathrm{em}}=$ $360 \mathrm{~nm}$. The system included a flow through needle (FTN) injection system with a $15 \mu \mathrm{L}$ needle. The dwell volume $\left(\mathrm{V}_{\mathrm{d}}\right)$ was experimentally measured as $110 \mu \mathrm{L}$. Data acquisition, data handling and instrument control were performed with Empower 2 (Waters).

IEX measurements were performed on a Waters ACQUITY UPLC ${ }^{\mathrm{TM}}$ system equipped with a binary solvent delivery pump, an autosampler and fluorescence detector (FL). The Waters 
ACQUITY system included a $5 \mu \mathrm{L}$ sample loop and a $2 \mu \mathrm{L}$ FL flow-cell. The loop was directly connected to the injection switching valve. The measured dwell volume was around $100 \mu \mathrm{L}$. Fluorescence detection was carried out at $\lambda_{\mathrm{ex}}=280 \mathrm{~nm}$ and $\lambda_{\mathrm{em}}=360 \mathrm{~nm}$. The acquisition rate and time constant were fixed at $10 \mathrm{~Hz}$ and $50 \mathrm{~ms}$, respectively. Data acquisition and instrument control were performed by Empower Pro 3 Software (Waters).

Used in this study were ACQUITY UPLC Glycoprotein BEH Amide $300 \AA 1.7 \mu \mathrm{m}(2.1 \mathrm{~mm}$ id x $150 \mathrm{~mm}$,) and ACQUITY UPLC Peptide BEH C18 $300 \AA 1.7 \mu \mathrm{m}(2.1 \mathrm{~mm}$ id x $150 \mathrm{~mm}$, ) columns, both obtained from Waters (Milford, MA, USA). The IEX columns were YMC BioPro SP-F (4.6 mm i.d. $100 \mathrm{~mm}, 5 \mu \mathrm{m})$ strong cation exchanger purchased from Stacroma (Reinach, Switzerland).

\subsection{Analysis of insulins}

In HILIC and RPLC, the mobile phase was $0.1 \%(\mathrm{v} / \mathrm{v})$ TFA in water (A) and $0.1 \%(\mathrm{v} / \mathrm{v})$ TFA in ACN (B). The optimized conditions included an isocratic elution at $80 \% \mathrm{~B}$ and $31 \% \mathrm{~B}$ in HILIC and RPLC modes, respectively. The flow rates were set at $0.5 \mathrm{~mL} / \mathrm{min}$ and $0.3 \mathrm{~mL} / \mathrm{min}$ in HILIC and RPLC, respectively. The column temperature was set at $50^{\circ} \mathrm{C}$ in both modes. All insulins were diluted at $100 \mu \mathrm{g} / \mathrm{mL}$ in a $1: 1(\mathrm{v} / \mathrm{v})$ mixture of water/ACN with $0.1 \% \mathrm{FA}$ for HILIC or in water RPLC analysis, respectively. All experiments with insulins were performed in duplicate, and the chromatograms were identical between the two successive runs.

Human insulin is composed of 51 amino acid residues. It is a dimer of A- and B-chains, linked together by disulfide bridges. Bovine insulin differs from human insulin in only three amino acid residues: the threonine at position $A 8$ and isoleucine at position $A 10$ in the human insulin sequence are replaced by two valine residues, while the $\mathrm{C}$-terminal asparagine of the B-chain of human insulin is replaced by alanine. Insulin Lispro is a fast acting insulin analogue in which the penultimate lysine and proline residues on the $\mathrm{C}$ terminal end of the B-chain are reversed. Insulin glulisine is a rapid-acting insulin analogue that differs from human insulin in that the amino acid asparagine at position B3 is replaced by lysine and the lysine in position B29 is replaced by glutamic acid. Insulin aspart is also a fastacting insulin analog where a single amino acid has been exchanged (proline in position B28 replaced by aspartic acid). Insulin Glargine is a long-acting basal insulin analog. It has a substitution of glycine for asparagine at position A21 and has two arginine residues added to the C-terminal position of B-chain, shifting the isoelectric point from 5.4 to 6.7 and limiting deamidation of asparagine. 


\subsection{Characterization of interferon $a-2 b$}

\subsubsection{Oxidation}

Interferon $\mathrm{a}-2 \mathrm{~b}$ (Intron $\mathrm{A}$ at $15 \mathrm{mioUl} / \mathrm{mL}$ ) was oxidized in the presence of $1 \%(\mathrm{v} / \mathrm{v})$ hydrogen peroxide. After $60 \mathrm{~min}$ of incubation at $30^{\circ} \mathrm{C}$, the oxidation reaction was quenched by adding a small amount of methionine to the solution.

\subsubsection{Reduction}

The intramolecular disulfide bridges of interferon $a-2 b$ were reduced by adding DTT (in tenfold excess molarity from a $100 \mathrm{mM}$ solution) to its formulation, followed by incubation at 30 ${ }^{\circ} \mathrm{C}$ for $60 \mathrm{~min}$.

\subsubsection{Chromatographic conditions}

Closely related proteins (interferon $\alpha-2 b$ and its oxidized and reduced forms) were separated using the same (generic) gradient conditions in both HILIC and RPLC. The optimized HILIC conditions were the following. The mobile phase was $0.1 \%(\mathrm{v} / \mathrm{v})$ TFA in water $(A)$ and $0.1 \%$ $(\mathrm{v} / \mathrm{v})$ TFA in $\mathrm{ACN}(\mathrm{B})$, and the gradient program was $85 \%$ to $77 \% \mathrm{~B}$ in $0.2 \mathrm{~min}$, then 77 to $76 \% \mathrm{~B}$ in $7.8 \mathrm{~min}$. The flow rate was set at $0.45 \mathrm{~mL} / \mathrm{min}$. In RPLC, the same mobile phase was employed, but the gradient ran from $35 \%$ to $60 \% \mathrm{~B}$ in $8 \mathrm{~min}$, at a flow rate of 0.45 $\mathrm{ml} / \mathrm{min}$. The column temperature was set at $60^{\circ} \mathrm{C}$ and the injected volume was $0.5 \mu \mathrm{L}$ in both modes. The native interferon $a-2 b$ was directly injected from commercial solution of Intron $A$ at 15 millions of international units per $\mathrm{mL}$ ( $\mathrm{mioUl} / \mathrm{ml})$.

All experiments with interferon $a-2 b$ were performed in duplicate, and the chromatograms were identical between the two successive runs.

\subsection{Characterization of Trastuzumab samples}

\subsubsection{IdeS digestion}

Trastuzumab $(10 \mu \mathrm{g})$ was mixed with 10 units of IdeS enzyme and diluted in appropriate volume of $10 \mathrm{mM}$ Tris buffer at $\mathrm{pH} 7.4$ to obtain a final concentration of $1 \mathrm{mg} / \mathrm{mL} \mathrm{mAb}$. The digestion was performed at $37^{\circ} \mathrm{C}$ for $30 \mathrm{~min}$.

\subsubsection{Reduction}

Trastuzumab solution at $1 \mathrm{mg} / \mathrm{mL}$ in water was reduced by adding DTT (in ten-fold excess molarity from a $100 \mathrm{mM}$ solution), followed by incubation at $37^{\circ} \mathrm{C}$ for $45 \mathrm{~min}$. 


\subsubsection{IdeS digestion + reduction}

DTT was added to the trastuzumab solution obtained after digestion with IdeS. Then the sample was incubated at $37^{\circ} \mathrm{C}$ for $45 \mathrm{~min}$.

\subsubsection{Deglycosylation with EndoS2}

For deglycosylation with EndoS2, $10 \mu \mathrm{g}$ of Trastuzumab were mixed with 10 units of EndoS2 enzyme and diluted in appropriate volume of $10 \mathrm{mM}$ Tris buffer at $\mathrm{pH} 7.4$ to obtained final concentration at $1 \mathrm{mg} / \mathrm{mL}$ of $\mathrm{mAbs}$. The deglycosylation was performed at $37^{\circ} \mathrm{C}$ for $30 \mathrm{~min}$. Then, the deglycosylated form was digested by IdeS and/or reduced following the same procedure as for the intact form.

\subsubsection{Chromatographic conditions}

For HILIC and RPLC modes, the mobile phase was $0.1 \%$ TFA $(v / v)$ in water $(A)$ and $0.1 \%$ $(\mathrm{v} / \mathrm{v})$ TFA in $\mathrm{ACN}(\mathrm{B})$. The column temperature was set at $80{ }^{\circ} \mathrm{C}$ and the injection volume was $0.5 \mu \mathrm{L}$. The optimized HILIC gradient was $85 \%$ to $72 \%$ B in $0.2 \mathrm{~min}$, then 72 to $64 \% \mathrm{~B}$ in $10 \mathrm{~min}$, at a flow rate at $0.45 \mathrm{~mL} / \mathrm{min}$. The RPLC gradient was the following: 30 to $38 \% \mathrm{~B}$ in $12 \mathrm{~min}$, at a flow rate of $0.40 \mathrm{~mL} / \mathrm{min}$.

For IEX separation, mobile phase "A" consisted of $10 \mathrm{mM}$ MES in water, while mobile phase "B" was $10 \mathrm{mM}$ MES in water containing $1 \mathrm{M} \mathrm{NaCl}$. The $\mathrm{pH}$ of both mobile phases was adjusted by adding $1 \mathrm{M} \mathrm{NaOH}$ solution to titrate $\mathrm{pH}$ to 5.7 . Generic conditions, previously developed for $\mathrm{mAbs}$ were applied [22]. At a flow rate of $0.6 \mathrm{~mL} / \mathrm{min}$, the gradient was run from 0 to $20 \% \mathrm{~B}$ in $20 \mathrm{~min}$. Mobile phase temperature was set at $30{ }^{\circ} \mathrm{C}$. The injected volume was $2 \mu \mathrm{L}$.

For column coupling experiments, two and three HILIC columns were coupled in series (15, 30 and $45 \mathrm{~cm}$ ). For two columns, the total gradient time was set to $15 \mathrm{~min}$ at $0.3 \mathrm{~mL} / \mathrm{min}$. For the experiments performed with three columns, a $22.5 \mathrm{~min}$ long gradient at $0.2 \mathrm{~mL} / \mathrm{min}$ was applied. (The flow rate had to be reduced when increasing the column length because of pressure limitations).

All experiments with trastuzumab were performed in duplicate, and the chromatograms were identical between the two successive runs.

\subsubsection{Peak capacity calculation}

The following equation was used to estimate the peak capacity based on peak widths at $4 \sigma$, corresponding to a resolution of $R s=1$ between consecutive peaks: 


$$
n_{c}=1+\frac{t_{g}}{1.7 \cdot w_{50 \%}}
$$

The peak width measured at half height was used in this study, since proteins generally are heterogeneous and related proteins elute often in the front or in the tail of the main peak. Therefore, measurements near the baseline of a chromatogram can often be misleading. 


\section{Results and discussion}

It was our objective to establish simple HILIC-based chromatographic methods that could be used for the characterization of protein biopharmaceuticals. In large part, these investigations have been made possible by a new sub- $2 \mu \mathrm{m}$ stationary phase constructed of wide-pore hybrid silica bonded with an amide ligand. This phase uniquely facilitates large biomolecule separations given that its pore network is of a sufficiently large average diameter so as to be accessible to and to not cause restricted diffusion of large structures. In addition, its amide ligand imparts high HILIC retentivity, so that high resolution protein separations can be performed with mobile phases containing relatively high water content (Figure S-1). Interestingly, HILIC methods with this new stationary phase have proven to be most effective with trifluoroacetic acid (TFA) ion pairing, rather than ammonium salts, like (ammonium formate) and formic acid (FA), that have been commonly used in small molecule or released glycan HILIC separations. Results confirming the importance of using TFA ion pairing are shown in Figure S-2 [2,7]. Along with enhancing peak shape and resolution, TFA ion pairing has been found to reduce the retention of proteins in a HILIC separation. It is proposed that the acidic condition imparted by the TFA ensures that acidic residues of the protein are fully protonated and thus present in their more hydrophobic state. In addition, the ion pairing of the TFA counter ion to basic residues ensures that cationic residues will also be separated in a more hydrophobic form. In this way, the retention mechanism of a protein on the HILIC stationary phase is more uniformly driven by its hydrophilic residues and modifications, which can be exploited to achieve orthogonality versus conventional separation techniques. Because its mobile phase system is the same as that classically used for protein RPLC, a HILIC mode separation can be interchanged with an RPLC mode separation. And since TFAmodified mobile phases are volatile, such a HILIC mode separation can be readily coupled to ESI-MS, just like RPLC (Figure S-3).

\subsection{Separation of human, bovine and synthetic insulins in HILIC and RPLC}

Among biopharmaceuticals, insulin ( $\mathrm{MW}$ of $\sim 5.8 \mathrm{kDa}$ ) is one of the oldest, yet it remains the primary treatment for diabetes. In addition to recombinant human insulin, which became commercially available in 1982 [22], a number of closely related analogues (synthetic or modified versions of endogenous human insulin) were developed to alter the duration of action and elicit drug responses across long, intermediate or fast time frames [23]. Interest in insulin analogues also extends beyond diabetes treatment. Indeed, due to the suspected performance enhancement properties of insulin and its analogues, they have been prohibited by the world anti-doping agency (WADA) [24]. It is therefore important for there to be methods able to screen insulins potentially used in sports doping. 
In the present study, 6 insulins possessing different primary structures (Table 1) were considered to illustrate the performance of HILIC vs. RPLC. Figure 1 displays chromatograms obtained from HILIC with the wide-pore amide-bonded stationary phase versus RPLC with an analogous C18 bonded phase. Because the dimensions and particle diameters of the tested columns were identical $(150 \times 2.1 \mathrm{~mm}, 1.7 \mu \mathrm{m})$, and since both experiments were conducted in an isocratic mode, the kinetic performance of the two separations can be directly compared. As shown, the peak widths observed in the HILIC separation were comparable to those observed in an RPLC separation. Interestingly, the selectivity and elution order were quite different between the two chromatographic modes but the latter was not strictly opposite. This behaviour is expected since it is the hydrophobic amino acid residues that contribute most to retention in an RPLC separation, while it is presumably the hydrophilic amino acid residues that drive retention under HILIC conditions. For example, human insulin (1) and insulin glulisine (4) were not separated under RPLC conditions, while a baseline separation of these two insulins was easily achieved under HILIC conditions. Close examination of these two insulins shows that their primary sequences only differ with respect to two amino acid residues (one asparagine replaced by lysine and one lysine replaced by glutamic acid). Since these modifications are related to the replacement of one hydrophilic amino acid for another, it is understandable that these two insulins are better discriminated in HILIC. On the contrary, the separation of human insulin (1) and insulin lispro (3) was better in RPLC vs. HILIC. These two insulins are very closely related, with only one order inversion of two amino acid residues (lysine and proline).

\subsection{Characterization of Interferon $a-2 b$ sample under HILIC and RPLC conditions}

Interferon $\alpha-2 b$ is a 165 amino acid therapeutic glycoprotein that has a molecular weight of $19.2 \mathrm{kDa}$. In this example, intact, reduced and hydrogen peroxide oxidized interferon $a-2 b$ samples were analyzed in HILIC and RPLC modes. The corresponding chromatograms are reported in Figure 2. In these chromatograms, a large peak was systematically eluted close to the column dead time in both modes. This peak corresponds to m-cresol, an antimicrobial preservative present in the protein formulation at a relatively high concentration $(1.5 \mathrm{mg} / \mathrm{mL})$. Because of its unique chemical structure, $\mathrm{m}$-cresol is detectable, despite the use of selective fluorescence detection at excitation and emission wavelengths of 280 and 360, respectively. This substance was slightly retained under the RPLC conditions employed here $\left(k_{\text {app }}<1\right)$, while it was not retained in HILIC (under the applied conditions).

As illustrated in Figure 2, the two oxidized forms of interferon $a-2 b$ eluted either before or after their native counterparts depending on whether the separation mode was RPLC (Figure 
2A) or HILIC (Figure 2B), respectively. This behavior is in agreement with expectations, since an oxidation with hydrogen peroxide chemically transforms the thioether group of one or several methionine residues (Interferon $a-2 b$ contains 5 methionine residues) into sulfoxide. Because a sulfoxide moiety is more polar than a thioether group, retention is reduced in RPLC mode, while it is increased in HILIC.

The retention behavior of the reduced forms of Interferon $a-2 b$ was the opposite of the oxidized forms. With this therapeutic protein, two reduced forms were observed after incubation with DTT; each resulting from the cleavage of disulfide bridges located between cysteine residues (interferon contains four cysteine amino acids residues in its primary amino acid sequence that are capable of creating disulfide bridges). In RPLC, it is well known that the retention of an unfolded protein (reduced protein) is higher compared to the intact protein. This behavior can be attributed to the fact that protein unfolding generally exposes hydrophobic amino acid residues such that they have greater opportunity to interact with the hydrophobic stationary phase of an RPLC separation. On the contrary, this effect would be expected to decrease retention under HILIC conditions.

This example again confirms the orthogonality between RPLC and HILIC for the analytical characterization of therapeutic proteins. However, it is also clear that the retention differences between reduced, oxidized and intact proteins were much more noticeable in RPLC vs. HILIC. Indeed, a relatively wide gradient from 35 to $60 \%$ ACN was required in RPLC, while almost isocratic conditions (77 to $76 \% \mathrm{ACN}$ ) were necessary to elute all the forms of Interferon $\alpha-2 b$ in HILIC. Due to the particular condition employed (namely a very shallow gradient), and the fact that the analyzed protein was quite large ( $19.2 \mathrm{kDa})$, band broadening was quite significant under the employed HILIC conditions. However, it is within reason to suggest that much of this band broadening is a consequence of using a shallow gradient, wherein there is limited capacity to compress the analyte band. This was particularly true for the more strongly retained peaks (intact and oxidized forms). In addition, because interferon is a glycoprotein, several glycoforms may have been partially separated in HILIC, leading to broadened peaks for intact and oxidized interferon $\alpha-2 b$. Based on these effects, separations with lower apparent resolution were observed between the two oxidized forms of Interferon $a-2 b$ when using HILIC vs. RPLC modes of separation (see Figure $2 B$ ).

\subsection{Evaluation of HILIC for trastuzumab characterization}

\subsubsection{Impact of injected volume and initial gradient conditions in HILIC}

Perhaps the biggest challenge that must be addressed in the implementation of HILIC is how to best perform a sample injection. It is often recommended that a sample be prepared for chromatography in a diluent that matches the initial mobile phase composition. This practice cannot be strictly obeyed when developing HILIC based methods for protein 
biopharmaceuticals, since many proteins precipitate from high acetonitrile mixtures when they are held at ambient conditions. While additives and co-solvents can be used in HILIC sample diluents to enhance protein solubility, it is more desirable to develop a minimalistic approach in which protein sample is directly injected from an aqueous solution. At the same time, it must be understood that the strong elutropic strength of water can negatively impact adsorption and retention.

To this end, we studied the volumetric loading of an aqueous, reduced trastuzumab sample, knowing that peak distortion from solvent effects would likely be observed under some conditions (Figure 3). As can be seen, peaks can show very significant distortion when injected in too large of an aqueous volume, such as $2 \mu \mathrm{L}$ (which actually corresponds to only $0.6 \%$ of the column volume). No matter, optimized aqueous injections can in fact be achieved by decreasing the injected volume to $0.1-0.2 \%$ of the column volume and adding a fast initial ramp ( $0.2 \mathrm{~min})$ that incorporates a high ACN (85\%) condition into the beginning of the method. Indeed, when the injected volume was decreased down to $0.5 \mu \mathrm{L}$, the fragments of the reduced trastuzumab eluted as sharp peaks. Finally, it is anticipated that the ability of a HILIC method to accommodate aqueous injections correlates to some extent with the dispersion of the sample injection apparatus. That the employed UHPLC instrumentation exhibits extraordinarily low dispersion (band spread of $7.5 \mu \mathrm{L}$ ) might mean that other instrumentation may be capable of slightly higher aqueous volumetric loads.

\subsubsection{Impact of mobile phase temperature in HILIC conditions}

An important issue related to RPLC of mAbs is that the adsorption process becomes especially problematic at mobile phase temperatures lower than $60 \stackrel{\circ}{ }$, to a point that very poor recoveries are observed at such temperatures $[25,26]$. Since its interaction mechanism is entirely different, it was of interest to study whether HILIC presented a similar phenomenon. As demonstrated elsewhere [26], on-column adsorption is more significant for intact $\mathrm{mAbs}$ and large fragments rather than small fragments, like a light chain subunit.

With this in mind, intact trastuzumab was injected at mobile phase temperatures of 40,60 and $80{ }^{\circ} \mathrm{C}$ in both RPLC and HILIC modes. With RPLC (Figure 4A), a peak area reduction of $15 \%$ was noticed in decreasing the mobile phase temperature from 80 to $60{ }^{\circ} \mathrm{C}$, and at 40 ${ }^{\circ} \mathrm{C}, 2 \%$ or less of the trastuzumab was recovered. This trend is in good agreement with previous findings $[25,26]$. In contrast, this recovery issue was much less pronounced in HILIC. Indeed, at 40 and $60{ }^{\circ} \mathrm{C}$, around $80 \%$ of the original peak area (observed at $80{ }^{\circ} \mathrm{C}$ ) was recovered. Even if recovery from stationary phases is mAb dependent [25], this first example on trastuzumab illustrates what may be one of the most important benefits of HILIC, namely the possibility to work at reasonable mobile phase temperatures, without appreciable 
sample loss. This characteristic is appealing since the use of high column temperatures (i.e. $80 \stackrel{\circ}{ } \mathrm{C})$ comes with an increased risk of on-column degradation.

\subsubsection{Analytical characterization of trastuzumab samples in HILIC, RPLC and IEX}

For the detailed characterization of a mAb, several orthogonal methods can be applied to gather a collection of information on its microheterogeneity (post-translational modifications and degradation products). For this reason, we have investigated the value of HILIC in a multifaceted chromatographic approach including both IEX and RPLC. IEX is commonly used for profiling the charge heterogeneity of mAbs resulting either from enzymatic or chemical modifications. Among the various IEX modes, cation-exchange (CEX) is considered to be the gold standard for the analysis of therapeutic proteins. In RPLC, protein variants are separated mainly on the basis of their hydrophobicity, but due to possible secondary interactions, some additional selectivity can also be expected. This chromatographic mode is mostly used to separate protein species possessing hydrophobicity differences (e.g. oxidized, reduced, degraded forms). Its popularity has increased over the years thanks to its high resolving power compared to IEX and inherent MS compatibility. Yet, now with the viability of methods shown above, HILIC too can be used in a strategy for thoroughly characterizing a mAb.

Using HILIC, RPLC and IEX, trastuzumab samples have been subjected to different levels of analysis, including so-called 'middle-up' samples prepared by enzymatic cleavage with the enzyme IdeS. IdeS, a protease that cleaves in the hinge region of humanized mAbs, is an increasingly popular tool for $\mathrm{mAb}$ characterization, given that it generates fragments more amenable than intact mAb samples to both chromatographic and mass spectrometric techniques. This sort of partial enzymatic digestion, just like reduction of disulfide bonds, can be used to facilitate an analysis (as illustrated in Figure S-4). Figure 5 presents chromatograms obtained for these samples by RPLC, HILIC and IEX. In RPLC, intact trastuzumab (blue chromatograms) eluted in one single, but not homogeneous, peak. Similarly, one peak was obtained in HILIC, but the large width of the peak again suggests a potential, partial separation of non-homogeneous species. The highest resolution for intact trastuzumab was achieved in IEX, where it was possible to resolve a few peaks at the front of the main species. Not surprisingly, peak capacities were estimated to be higher for intact trastuzumab with the IEX separation, since several species were separated, leading to a narrower peak (Table 2). 
Light chain (LC) and heavy chain ( $\mathrm{HC}$ ) fragments of trastuzumab were, on the other hand, well separated from each other in both RPLC and HILIC, but not in IEX (orange chromatograms in Figures 5 and 6). However, an important distinction between the RPLC and HILIC separations was observed. In RPLC, only one peak was observed for the HC, but by HILIC, the HC was separated into at least four peaks. These four peaks correspond to glycovariants of the $\mathrm{HC}$, which contains the conserved mAb N-glycosylation site. As identified by separate HILIC-MS analyses, these peaks can be attributed to the major $\mathrm{N}$ glycan structures of trastuzumab (G0, G0F, G1F, and G2F) (Figure S-5) [27].

To better support the suggestion that HILIC and not RPLC produces glycoform separations, experiments were also performed on deglycosylated trastuzumab (Figure 6). The chromatographic profiles, retention orders and peak widths in RPLC (figures 5A and 6A) remained strictly identical to the non-deglycosylated trastuzumab samples, thus confirming that the separation of glycoforms cannot be performed with this separation mode. In HILIC, the retention times of the LC and $F(a b)^{\prime} 2$ subunits were not affected by deglycosylation (Figures 5B and $6 \mathrm{~B}$ ), as these fragments do not contain $\mathrm{N}$-glycosylation sites. On the contrary, the retention windows of the intact, $\mathrm{HC}$ and $\mathrm{Fc} / 2$ species shifted from 7.8 - 8 min, 5.3 - $6.3 \mathrm{~min}$ and $4.5-5.5 \mathrm{~min}$ to a lower retention of $6.2-6.8,4-4.5$ and $2.8-3.1 \mathrm{~min}$, when considering the native trastuzumab vs deglycosylated trastuzumab samples. Moreover, the HILIC peak for deglycosylated, intact trastuzumab was observed to be sharper (Figure $6 \mathrm{~B}$ ) versus native trastuzumab (Figure $5 \mathrm{~B}$ ) as the separation was no longer challenged by the incomplete resolution of glycoforms. Both the $\mathrm{HC}$ and $\mathrm{Fc} / 2$ of the deglycosylated $\mathrm{mAb}$ eluted in one single peak, while the $\mathrm{HC}$ and $\mathrm{Fc} / 2$ of the non-deglycosylated mAb eluted in several peaks. Since the intact trastuzumab as well as the $\mathrm{HC}$ and $\mathrm{Fc} / 2$ fragments contain the $\mathrm{N}$-glycosylation sites of trastuzumab, it is logical to conclude that these HILIC conditions allow for the differentiation of mAb glycoforms at the intact and middle-up levels of analysis. This is a powerful feature as MS spectra could be simplified when analyzing large fragments of mAbs in HILIC-MS vs. RPLC-MS.

Similar conclusions were drawn from the IdeS digested sample (black chromatograms in Figures 5 and 6). An IdeS digest of trastuzumab produces one subunit, the Fc/2, containing the canonical mAb glycosylation site. The fact that the $\mathrm{Fc} / 2$ fragment eluted as numerous peaks under HILIC conditions, but only as one peak in RPLC, again shows that HILIC is capable of resolving protein glycoforms. The chromatograms obtained for the reduced/ldeS digested sample (yellow chromatograms in Figures 5 and 6), presented a similar case of glycoforms being directly profiled by HILIC at the middle-up level of analysis. Results obtained from hyphenating this technique with MS have indeed confirmed that the heterogeneity observed by HILIC can be attributed to resolving Fc/2 subunits containing different $\mathrm{N}$-glycan structures, including lower abundance afucosylated G0 and G1 species 
(Figure S-6). It must also be mentioned that in application to profiling reduced, IdeS digested trastuzumab, IEX (Figure 5C) proved to be less informative. Evidence of sample heterogeneity was observed but only with poor resolution. Clearly then, HILIC is complementary to alternative separation techniques. Importantly, it shows the unique ability to be used to chromatographically differentiate glycoforms. In addition, and as demonstrated in this work, HILIC can be easily combined with MS such that the glycoforms of intact mAbs or their large fragments can be more thoroughly dissected through the generation of cleaner, simplified MS spectra.

\subsubsection{Improving separation power by increasing column length}

Looking to further enhance these new HILIC separations, we exploited longer column lengths to improve peak capacity. Chromatograms corresponding to the use of coupled columns are reported in Figure 7.

When separated on a $150 \mathrm{~mm}$ long column, intact trastuzumab appeared as a homogeneous and reasonably symmetrical peak. With an increased column length of $300 \mathrm{~mm}$, some additional species were observed under the main peak. With a $450 \mathrm{~mm}$ long column, it became clearer that the intact trastuzumab is not homogeneous, and that it might be represented by numerous species with very similar retention times. Whatever the column length, the current performance achieved in HILIC for intact trastuzumab is too limited to draw solid conclusions, but it is reasonable to speculate that the $450 \mathrm{~mm}$ column produced a partial separation of the main glycoforms of trastuzumab that have previously been reported in the literature, including G0/G0F, G0F/G0F, G0F/G1F, G0F/G2F and G1F/G1F [27].

Separations of the reduced/IdeS digested sample on increasingly longer column lengths proved to be equally interesting (Figure 6B). Peak capacity was found to increase from 108 to 141 to 183 on 150,300 and $450 \mathrm{~mm}$ long columns, respectively. These sizable gains in resolving power led to an improvement in resolution, particularly in the resolution of the $\mathrm{Fc} / 2$ glycoforms. In brief, applying long columns in HILIC appears to be an interesting strategy that is made possible by the low viscosity of HILIC mobile phases. The HILIC conditions developed in this work easily facilitate the use of long (up to $450 \mathrm{~mm}$ ) column lengths on commercial UHPLC systems. 


\section{Conclusion}

In this work, HILIC separations were developed for the characterization of biopharmaceuticals at the intact protein and middle-up levels of analysis. A new HILIC stationary phase based on amide-bonded, wide-pore hybrid silica facilitated investigations into these novel applications of HILIC. This material was successfully used for the analysis of diverse insulins $(\sim 6 \mathrm{kDa})$, interferon $\mathrm{a}-2 \mathrm{~b}(\sim 19 \mathrm{kDa})$ as well as trastuzumab $(\sim 150 \mathrm{kDa})$ and its large fragments $(\sim 25-100 \mathrm{kDa})$. The HILIC mobile phase consisted of $65-80 \% \mathrm{ACN}$ and $0.1 \%$ TFA was systematically added to both water and ACN to improve peak shapes while achieving reasonable retention. In addition, totally aqueous protein samples were successfully analyzed under HILIC conditions, showing that with appropriate method considerations, HILIC sample injections can be simplified. HILIC and RPLC separations of the same protein samples were seen to be quite orthogonal in terms of elution order and selectivity, while the kinetic performance remains comparable. This behavior can be explained, since RPLC differentiates proteins mostly via their hydrophobic amino acid residues, while HILIC separates protein species based on their hydrophilic amino acid residues and glycans. HILIC analysis of trastuzumab at the intact protein and middle-up levels proved to be particularly attractive since glycovariants were separated, where they could not be separated by RPLC or IEX. Several additional benefits were also noticed when using HILIC conditions to characterize protein biopharmaceuticals, such as: i) its inherent compatibility with MS, which is not the case of IEX, ii) no requirement for elevated mobile phase temperatures (which are often in the range $80-90^{\circ} \mathrm{C}$ in RPLC to improve protein recovery) and iii) the possibility to couple several HILIC columns in series to further improve resolving power, thanks to the limited viscosity of the highly organic mobile phase. Nevertheless, a strong peak distortion phenomenon was sometimes observed with HILIC conditions. This behaviour was, however, minimized through the use of a low injection volume (aqueous volume to be less than $0.5 \%$ of the column volume), and a fast gradient step ( $0.2 \mathrm{~min})$ to allow for relatively high ACN (85\%) loading conditions at the beginning of the gradient. In sum, HILIC shows significant promise as a chromatographic technique for the characterization of protein biopharmaceuticals such that it is foreseeable for it to become as effective a tool as RPLC and IEX. Finally, in looking toward future method development, it could be suggested that wide-pore superficially porous particles (SPP) could be a means to further improving the kinetic performance of these new, large molecule HILIC separations. 


\section{Acknowledgements}

The authors gratefully acknowledge Marleen van Wingerden and Bruno Morain from Waters for the scientific discussions and exchange of ideas on this new wide-pore HILIC material. The authors are also grateful to Dr. Raul Nicoli (Swiss Antidoping laboratory, Epalinges, Switzerland) for providing the human and synthetic insulin samples used in this study. Davy Guillarme and Jean-Luc Veuthey wish to thank the Swiss National Science Foundation for support through a fellowship to Szabolcs Fekete (31003A 159494). 


\section{References}

[1] S. Tandon, S. Sharma, R. Rajput, P.K. Yadav, K. Singh, Biotech Drugs: The next boom in pharmaceutical market, J. Pharm. Res. Opin. 1 (2011) 76-79.

[2] S. Fekete, D. Guillarme, Ultra-high-performance liquid chromatography for the characterization of therapeutic proteins, Trends Anal. Chem. 63 (2014) 76-84.

[3] M. Swartz, I.S. Krull, Analytical Method Validation for Biotechnology Proteins, Peptides, and Antibodies, LC-GC N. A. 27 (2009) 550-567.

[4] S. Fekete, D. Guillarme, Reversed-phase liquid chromatography for the analysis of therapeutic proteins and recombinant monoclonal antibodies, LC-GC Eur. 25 (2012) 540550.

[5] W.R. Strohl, D.M. Knight, Discovery and development of biopharmaceuticals: current issues, Curr. Opin. Biotechnol. 20 (2009) 668-672.

[6] A. Staub, D. Guillarme, J. Schappler, J.L. Veuthey, S. Rudaz, Intact protein analysis in the biopharmaceutical field, J. Pharm. Biomed. Anal. 55 (2011) 810-822.

[7] S. Fekete, D. Guillarme, P. Sandra, K. Sandra, Chromatographic, Electrophoretic and Mass Spectrometric Methods for the Analytical Characterization of Protein Biopharmaceuticals, Anal. Chem. 88 (2016) 480-507.

[8] A.S. Rathore, Follow-on protein products: scientific issues, developments and challenges, Trends Biotech. 27 (2009) 698-705.

[9] B. Bobaly, A. Beck, J. Fekete, D. Guillarme, S. Fekete, Systematic evaluation of mobile phase additives for the LC-MS characterization of therapeutic proteins, Talanta 136 (2015) 60-67.

[10] B. A. Rogers, Z. Wu, B. Wei, X. Zhang, X. Cao, O. Alabi, M. J. Wirth, Submicrometer Particles and Slip Flow in Liquid Chromatography, Anal. Chem., 87 (2015) 2520-2526.

[11] A. Periat, J. Boccard, J.L. Veuthey, S. Rudaz, D. Guillarme, Systematic comparison of sensitivity between hydrophilic interaction liquid chromatography and reversed phase liquid chromatography coupled with mass spectrometry, J. Chromatogr. A 1312 (2013) 49-57.

[12] H.P. Nguyen, K.A. Schug, The advantages of ESI-MS detection in conjunction with HILIC mode separations: Fundamentals and applications, J. Sep. Sci. 31 (2008) 1465-1480.

[13] B. Buszewski, S. Noga, Hydrophilic interaction liquid chromatography (HILIC)—a powerful separation technique, Anal. Bioanal. Chem. 402 (2012) 231-247.

[14] M.R. Gama, R.G. Da Costa Silva, C.H. Collins, C.B.G. Bottoli, Hydrophilic interaction chromatography, Trends Anal. Chem. 37 (2012) 48-60.

[15] R.I. Chirita, C. West, A.L. Finaru, C. Elfakir, Approach to hydrophilic interaction chromatography column selection: Application to neurotransmitters analysis, J. Chromatogr. A 1217 (2010) 3091-3104. 
[16] J. Ruta, J. Boccard, D. Cabooter, S. Rudaz, G. Desmet, J.L. Veuthey, D. Guillarme, Method development for pharmaceutics: some solutions for tuning selectivity in reversed phase and hydrophilic interaction liquid chromatography, J. Pharm. Biomed. Anal. 63 (2012) 95-105.

[17] D.V. McCalley, Is hydrophilic interaction chromatography with silica columns a viable alternative to reversed-phase liquid chromatography for the analysis of ionisable compounds?, J. Chromatogr. A 1171 (2007) 46-55.

[18] A. Periat, I.S. Krull, D. Guillarme, Applications of hydrophilic interaction chromatography to amino acids, peptides, and proteins, J. Sep. Sci. 38 (2015) 357-367.

[19] J. Carroll, L.M. Fearnley, J.E. Walker, Definition of the mitochondrial proteome by measurement of molecular masses of membrane proteins, Proc. Natl. Acad. Sci. U. S. A. 103 (2006) 16170-16175.

[20] T. Tetaz, S. Detzner, A. Friedlein, B. Molitor, J.L. Mary, Hydrophilic interaction chromatography of intact, soluble proteins, J. Chromatogr. A 1218 (2011) 5892-5896.

[21] A. Pedrali, S. Tengattini, G. Marrubini, T. Bavaro, P. Hemstrom, G. Massolini, M. Terreni, C. Temporini, Characterization of Intact Neo-Glycoproteins by Hydrophilic Interaction Liquid Chromatography, Molecules 19 (2014) 9070-9088.

[22] K. Sandra, I. Vandenheede, P. Sandra, Modern chromatographic and mass spectrometric techniques for protein biopharmaceutical characterization, J. Chromatogr. A 1335 (2014) 81-103.

[23] E.E. Chambers, C. Legido-Quigley, N. Smith, K.J. Fountain, Development of a fast method for direct analysis of intact synthetic insulins in human plasma: the large peptide challenge, Bioanalysis 5 (2013) 65-81.

[24] R. Nicoli, D. Guillarme, N. Leuenberger, N. Baume, N. Robinson, M. Saugy, J.L. Veuthey, Analytical strategies for doping control purposes: needs, challenges and perspectives, Anal. Chem. 88 (2016) 508-523.

[25] S. Fekete, A. Beck, E. Wagner, K. Vuignier, D. Guillarme, Adsorption and recovery issues of recombinant monoclonal antibodies in reversed-phase liquid chromatography, J. Sep. Sci. 38 (2015) 1-8.

[26] S. Fekete, S. Rudaz, J.L. Veuthey, D. Guillarme, Impact of mobile phase temperature on recovery and stability of monoclonal antibodies using recent reversed phase stationary phases, J. Sep. Sci. 35 (2012) 3113-3123.

[27] A. Beck, F. Debaene, H. Diemer, E. Wagner-Rousset, O. Colas, A. Van Dorsselaer, S. Cianferani, Cutting-edge mass spectrometry characterization of originator, biosimilar and biobetter antibodies, J. Mass Spectrom. 50 (2015) 285-297. 


\section{Figure captions}

Figure 1: Separation of human, bovine and synthetic insulins in: (A) RPLC-UV conditions and (B) HILIC-UV conditions. Peak identification: (1) Human insulin, (2) Bovine insulin, (3) Insulin Lispro, (4) Insulin glulisine, (5) Insulin aspart, (6) Insulin glargine. For both RPLC and HILIC, the mobile phase was $0.1 \%(\mathrm{v} / \mathrm{v})$ TFA in water $(\mathrm{A})$ and $0.1 \%(\mathrm{v} / \mathrm{v})$ TFA in ACN (B). RPLC conditions: ACQUITY UPLC Peptide BEH C18 $300 \AA 1.7 \mu \mathrm{m}(2.1 \mathrm{~mm}$ id x $150 \mathrm{~mm})$ column, isocratic elution at $31 \% \mathrm{~B}$ and a flow rate of $0.3 \mathrm{~mL} / \mathrm{min}$. HILIC conditions: ACQUITY UPLC Glycoprotein BEH Amide $300 \AA 1.7 \mu \mathrm{m}(2.1 \mathrm{~mm}$ id x $150 \mathrm{~mm})$ column, isocratic elution at 80 $\% B$ and a flow rate of $0.5 \mathrm{~mL} / \mathrm{min}$. The mobile phase temperature was set at $50^{\circ} \mathrm{C}$ for both separation modes.

Figure 2: Analysis of intact, oxidized and reduced interferon $a-2 b$ samples in (A) RPLC-FD conditions and (B) HILIC-FD conditions. For both RPLC and HILIC, the mobile phase was $0.1 \%(\mathrm{v} / \mathrm{v})$ TFA in water $(\mathrm{A})$ and $0.1 \%(\mathrm{v} / \mathrm{v})$ TFA in ACN (B). RPLC conditions: ACQUITY UPLC Peptide BEH C18 $300 \AA 1.7 \mu \mathrm{m}(2.1 \mathrm{~mm}$ id x $150 \mathrm{~mm}$ ) column, 35\% to 60\% B in $8 \mathrm{~min}$ and flow rate at $0.45 \mathrm{ml} / \mathrm{min}$. The mobile phase temperature was set at $60^{\circ} \mathrm{C}$. HILIC conditions: ACQUITY UPLC Glycoprotein BEH Amide $300 \AA 1.7 \mu \mathrm{m}(2.1 \mathrm{~mm}$ id x $150 \mathrm{~mm})$ column, $85 \%$ to $77 \% \mathrm{~B}$ in $0.2 \mathrm{~min}$, then 77 to $76 \% \mathrm{~B}$ in $7.8 \mathrm{~min}$. The flow rate was set at 0.45 $\mathrm{mL} / \mathrm{min}$. The mobile phase temperature was set at $60^{\circ} \mathrm{C}$.

Figure 3: Impact of injected volume for reduced trastuzumab separated under HILIC conditions, when adding a fast initial ramp to the gradient program. Chromatographic conditions: ACQUITY UPLC Glycoprotein BEH Amide $300 \AA 1.7 \mu \mathrm{m}(2.1 \mathrm{~mm}$ id x $150 \mathrm{~mm})$ column, $85 \%$ to $72 \% \mathrm{~B}$ in $0.2 \mathrm{~min}$, then 72 to $64 \% \mathrm{~B}$ in $10 \mathrm{~min}$, and a flow rate of 0.45 $\mathrm{mL} / \mathrm{min}$. The mobile phase temperature was set at $80^{\circ} \mathrm{C}$. The injection volume was varied between 0.5 and $2 \mu \mathrm{L}$.

Figure 4: Impact of mobile phase temperature on the recovery of intact trastuzumab in $(A)$ RPLC and (B) HILIC conditions. RPLC conditions: ACQUITY UPLC Peptide BEH C18 $300 \AA$ $1.7 \mu \mathrm{m}(2.1 \mathrm{~mm}$ id $\times 150 \mathrm{~mm})$ column, 30 to $38 \% \mathrm{~B}$ in $12 \mathrm{~min}$, and a flow rate of $0.40 \mathrm{~mL} / \mathrm{min}$. HILIC conditions: ACQUITY UPLC Glycoprotein BEH Amide $300 \AA 1.7 \mu \mathrm{m}(2.1 \mathrm{~mm}$ id x 150 $\mathrm{mm}$ ) column, $85 \%$ to $72 \% \mathrm{~B}$ in $0.2 \mathrm{~min}$, then 72 to $64 \% \mathrm{~B}$ in $10 \mathrm{~min}$, and a flow rate of 0.45 $\mathrm{mL} / \mathrm{min}$. The mobile phase temperature was set at 40,60 and $80^{\circ} \mathrm{C}$. 
Figure 5: Analysis of trastuzumab samples at the intact and middle-up levels of analysis using (A) RPLC, (B) HILIC and (C) IEX and wide-pore stationary phases. For RPLC and HILIC, the conditions are identical as in Figure 4, except that mobile phase temperature was set at $80{ }^{\circ} \mathrm{C}$. For IEX, a YMC BioPro SP-F (4.6 mm i.d. $\left.100 \mathrm{~mm}, 5 \mu \mathrm{m}\right)$ strong cation exchanger column was used. Mobile phase "A" consisted of $10 \mathrm{mM}$ MES in water, mobile phase " $\mathrm{B}$ " contained $10 \mathrm{mM}$ MES in water and $1 \mathrm{M} \mathrm{NaCl}(\mathrm{pH}=5.7)$. The gradient was run from 0 to $20 \%$ B in $20 \mathrm{~min}$ at a flow rate of $0.6 \mathrm{~mL} / \mathrm{min}$. Mobile phase temperature was set at $30{ }^{\circ} \mathrm{C}$.

Figure 6: Deglycosylated trastuzumab samples analyzed in (A) RPLC, (B) HILIC and (C) IEX. See the conditions as described in the caption of Figure 5.

Figure 7: Column coupling strategy for analyzing (A) intact trastuzumab and (B) IdeS digested/reduced trastuzumab under HILIC conditions. Columns of $150 \mathrm{~mm}$ lengths were used to obtain 150, 300 and $450 \mathrm{~mm}$ lengths. For one column, the gradient time was set at $10 \mathrm{~min}$, at a flow rate at $0.45 \mathrm{~mL} / \mathrm{min}$. For two columns, the total gradient time was set to 15 $\min$ at $0.3 \mathrm{~mL} / \mathrm{min}$. For the experiments performed with three columns, a $22.5 \mathrm{~min}$ long gradient at $0.2 \mathrm{~mL} / \mathrm{min}$ was applied. (The flow rate had to be reduced when increasing the column length because of pressure limitations). 

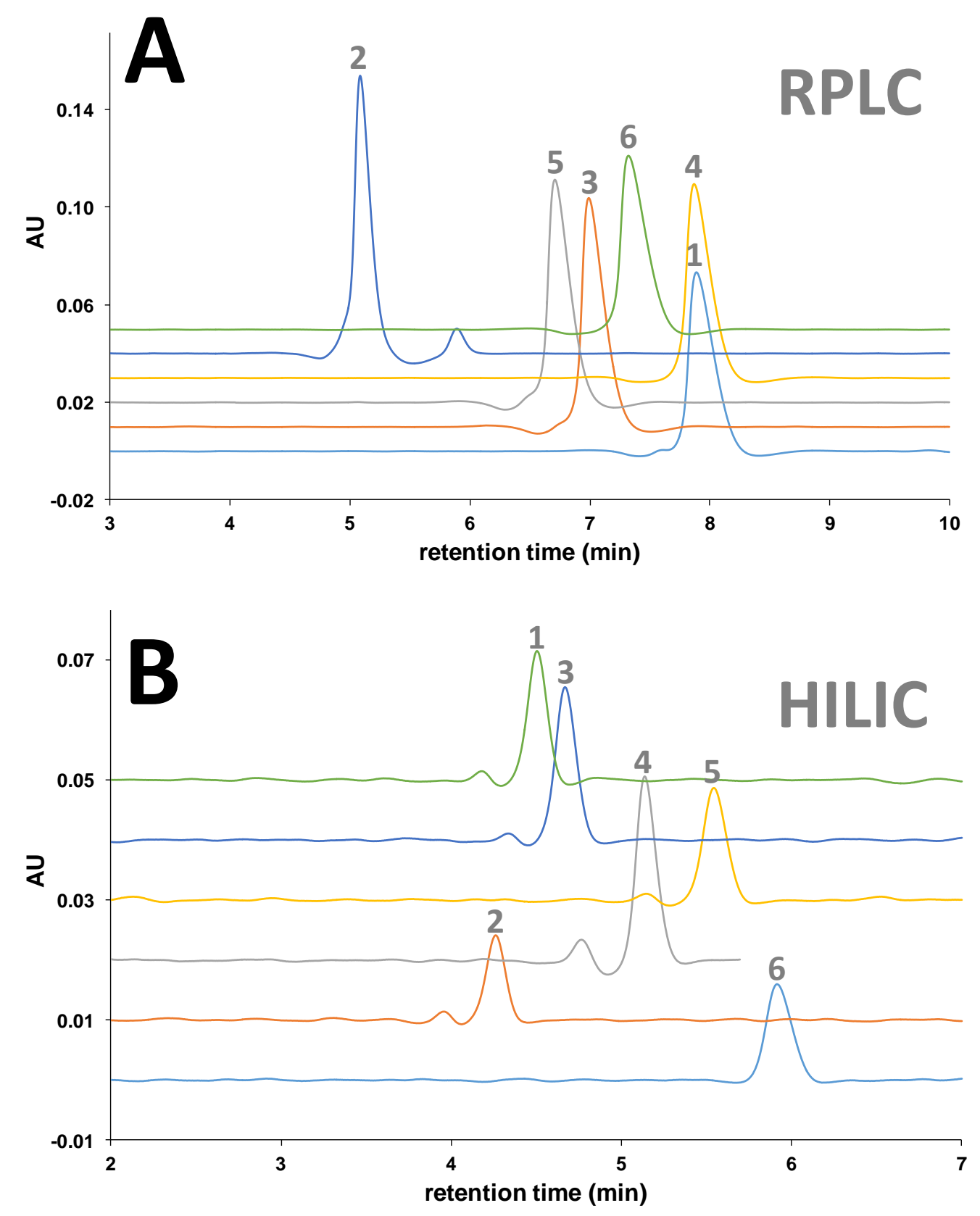

Figure 1 

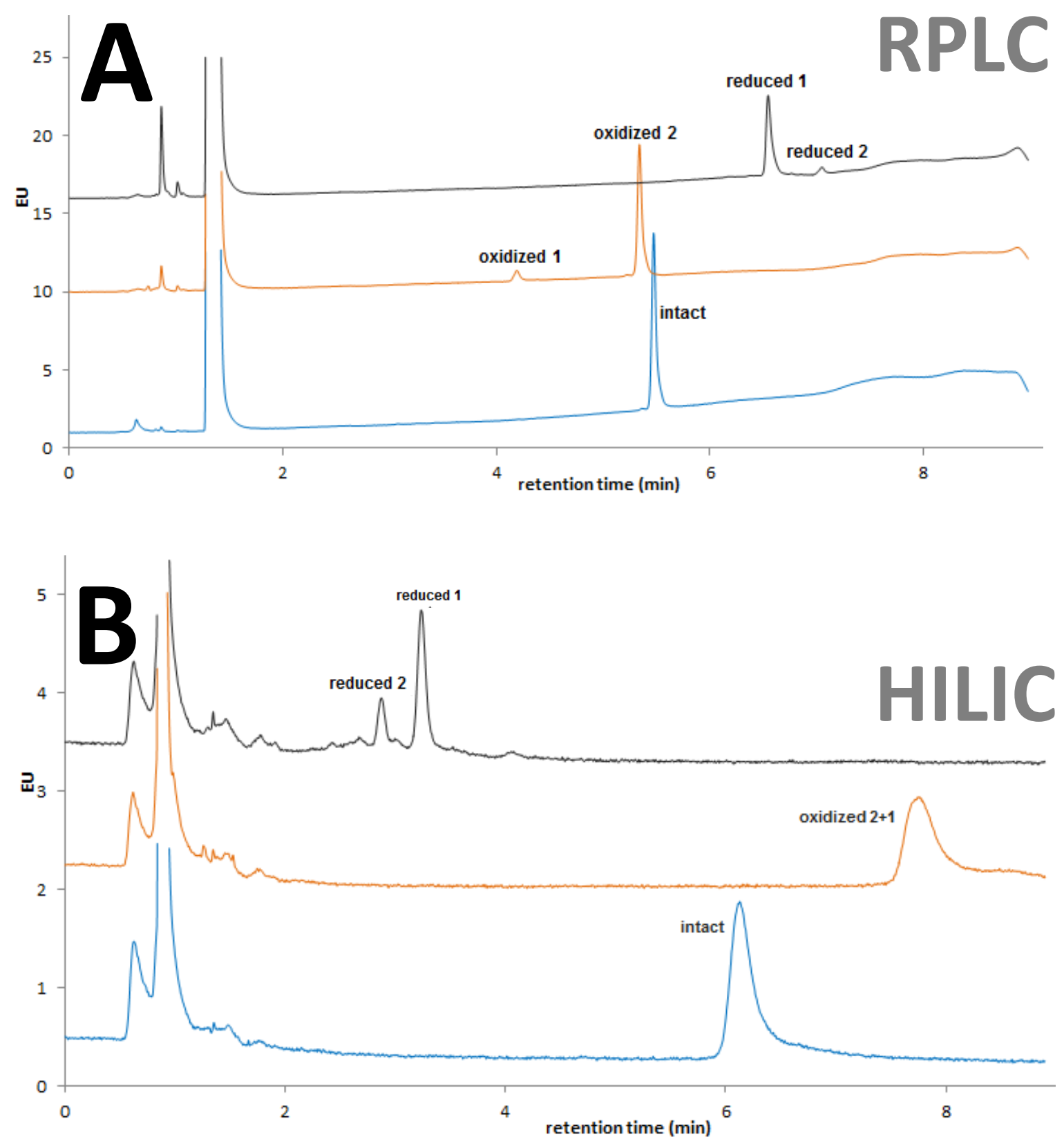

Figure 2 


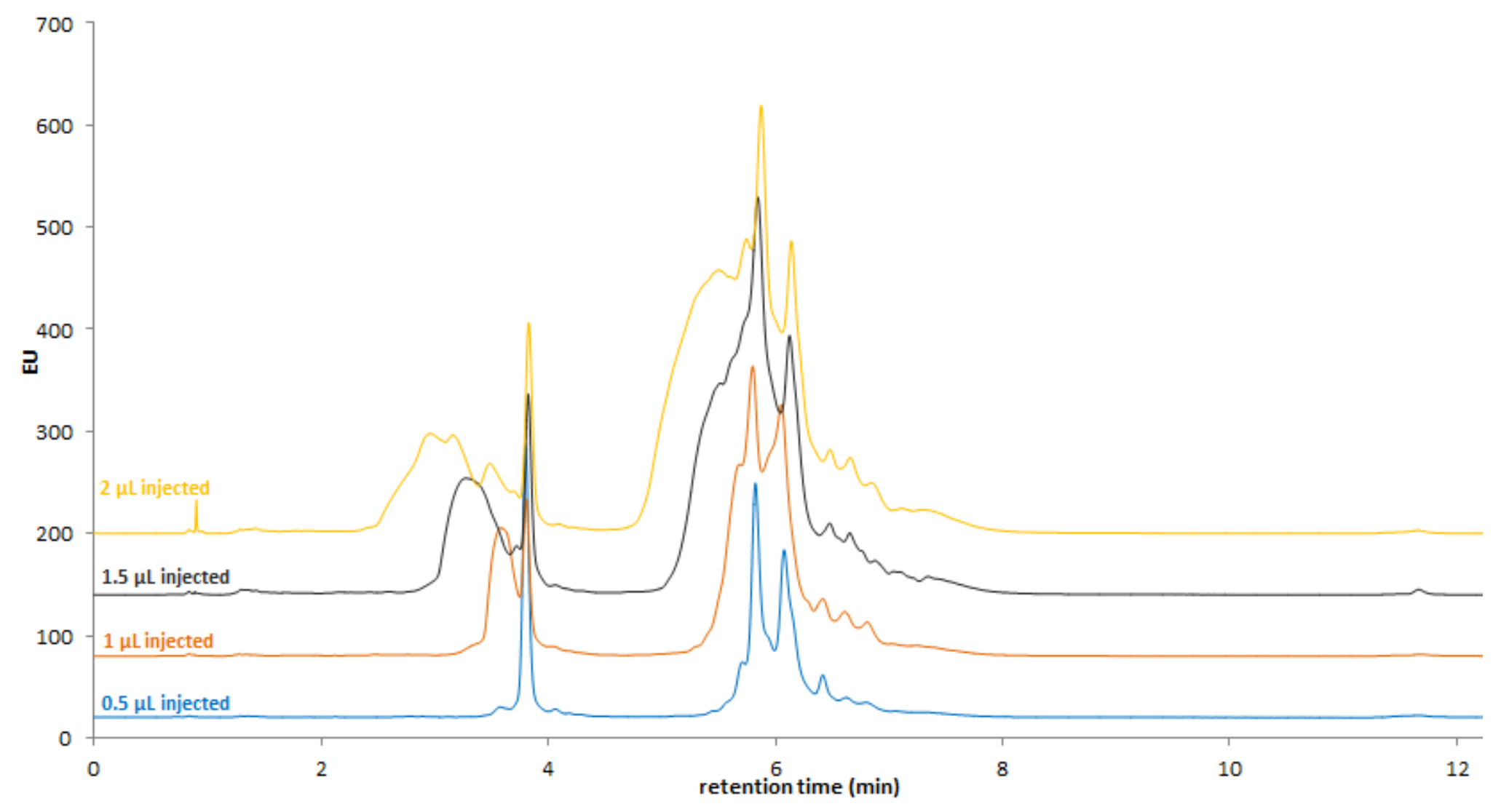

Figure 3 

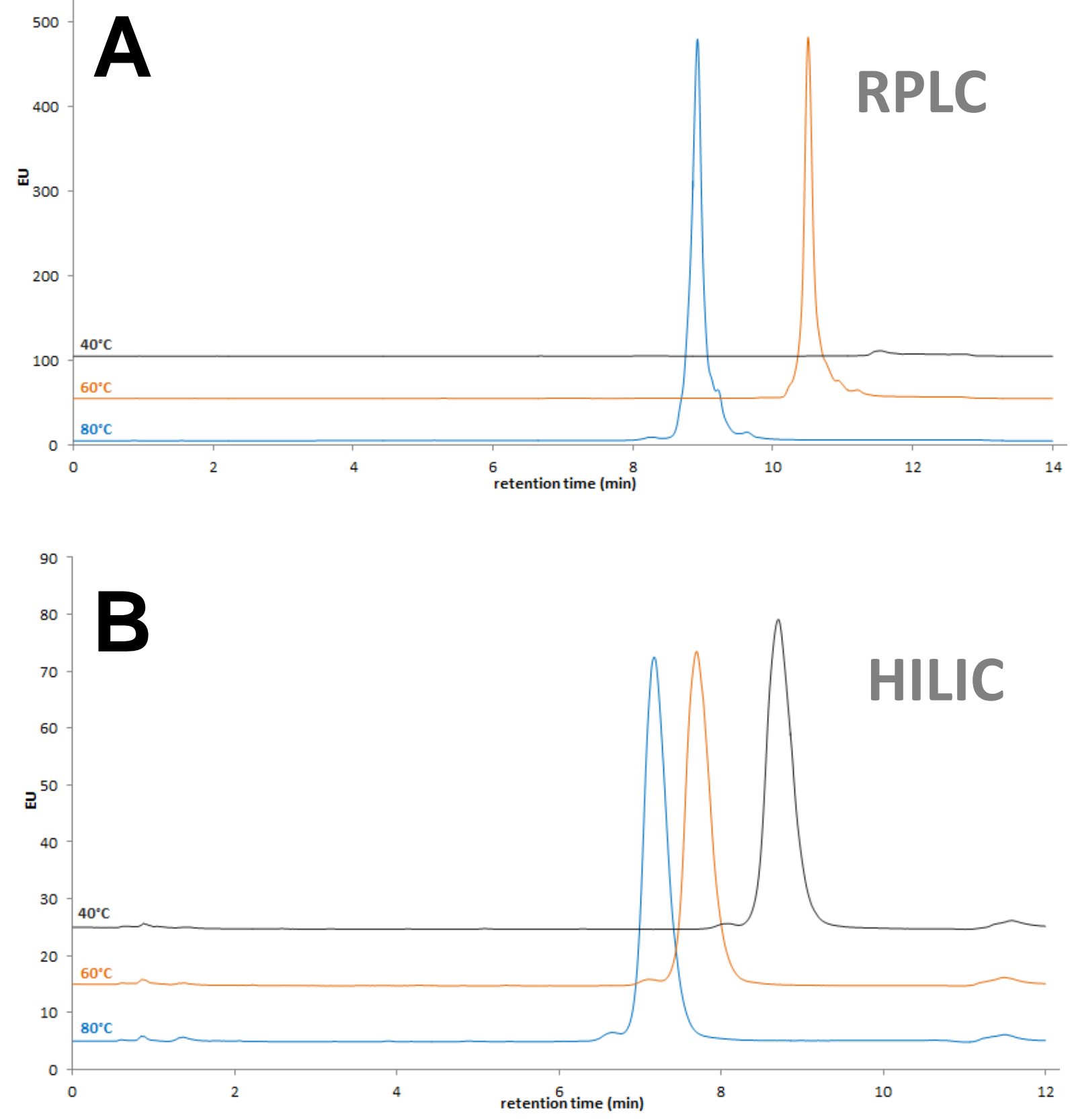

Figure 4 

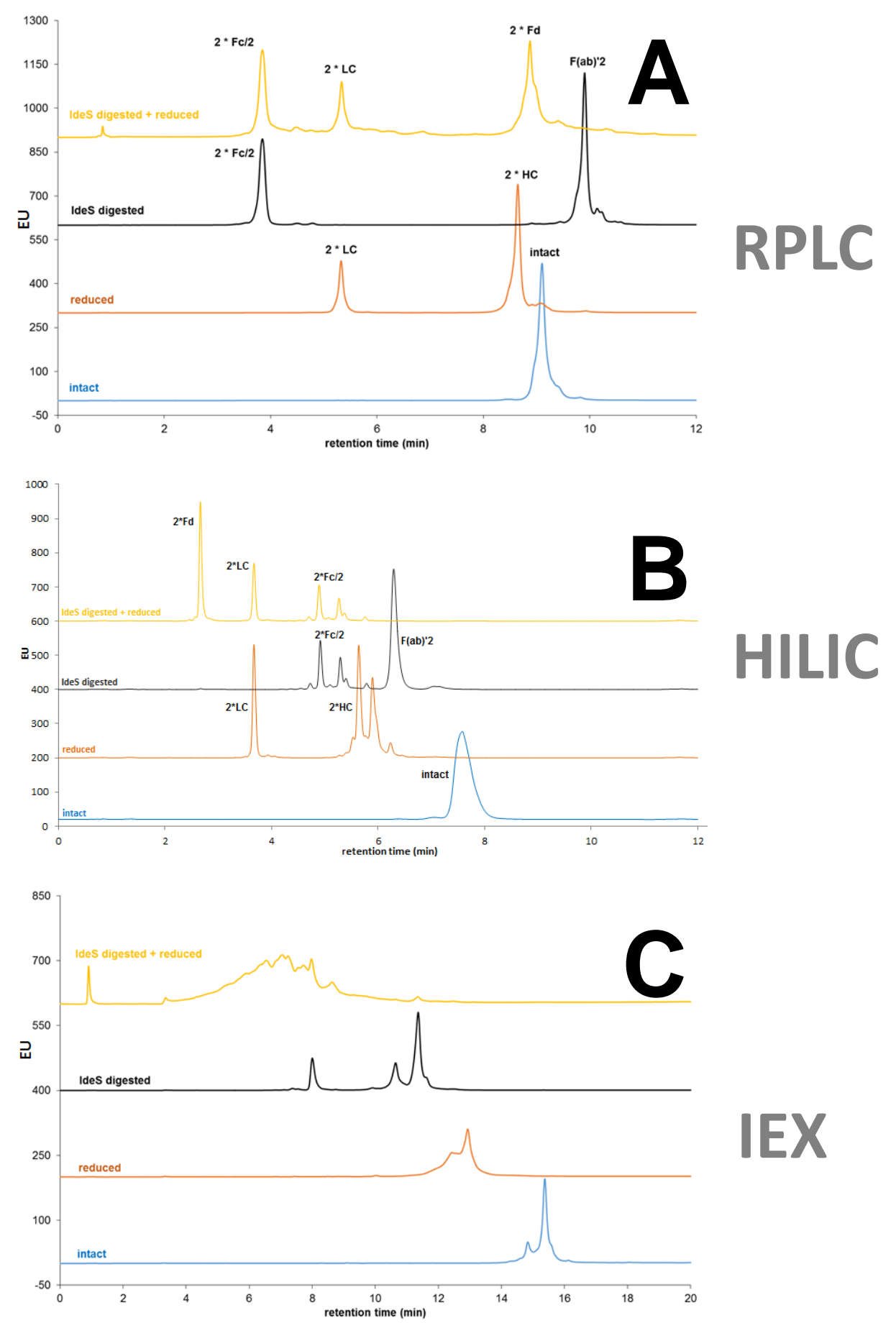

Figure 5 

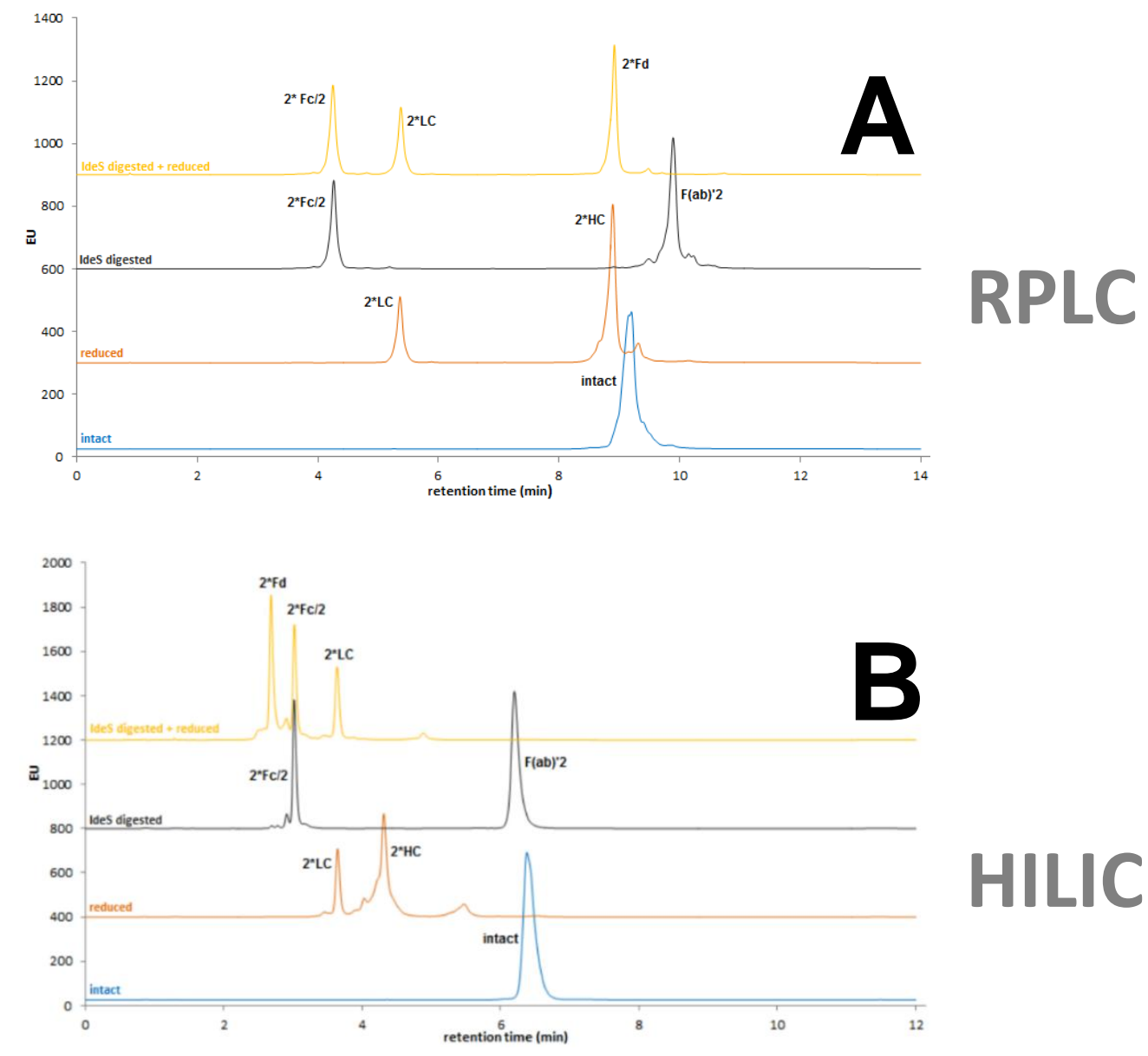

\section{HILIC}

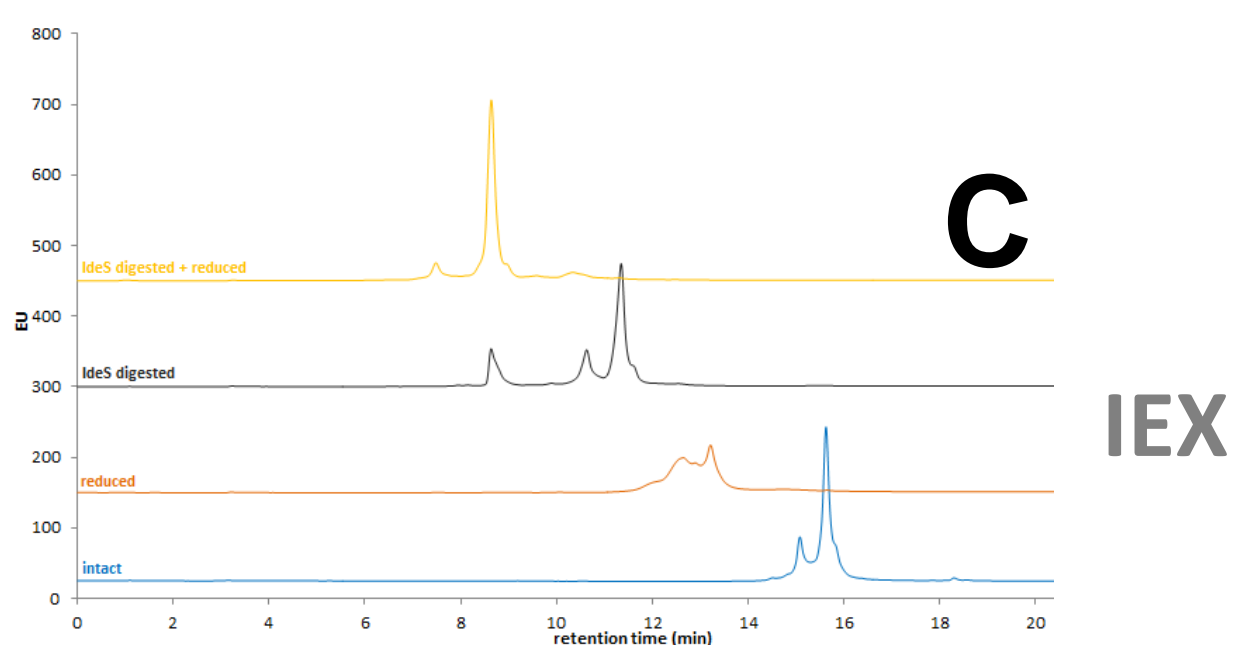

Figure 6 

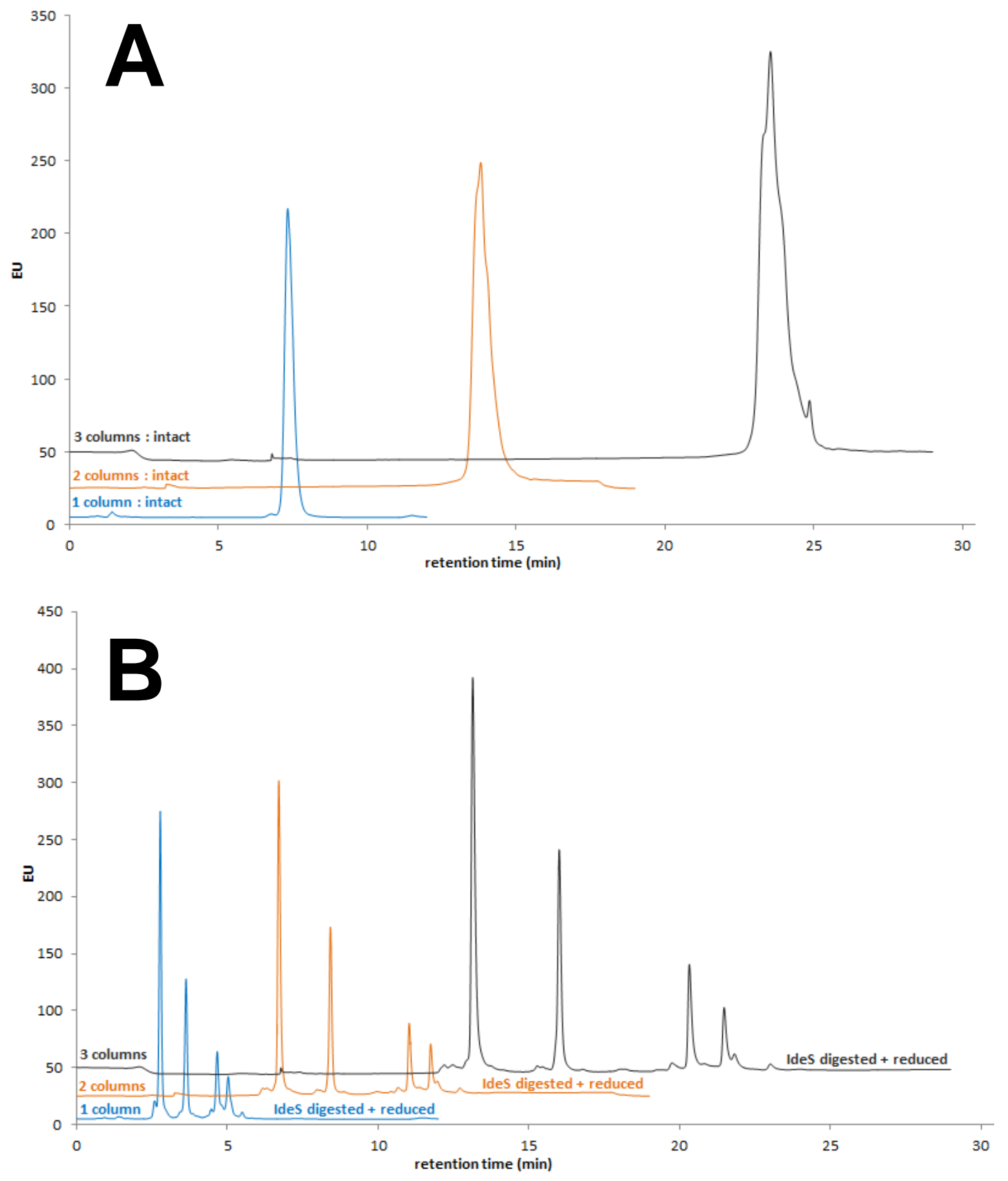

Figure 7 
Table 1. Properties of human, bovine and synthetic insulins. In the insulin structures, the $A$ and $B$ chains are linked through disulfide bridges.

\begin{tabular}{ccccc}
\hline Name & $\begin{array}{c}\text { Chemical } \\
\text { formula }\end{array}$ & MW (Da) & A chain sequence & B chain sequence \\
\hline Human insulin (1) & $\mathrm{C}_{257} \mathrm{H}_{383} \mathrm{~N}_{65} \mathrm{O}_{77} \mathrm{~S}_{6}$ & 5808 & GIVEQCCTSICSLYQLENYCN & FVNQHLCGSHLVEALYLVCGERGFFYTPKT \\
Bovine insulin (2) & $\mathrm{C}_{254} \mathrm{H}_{377} \mathrm{~N}_{65} \mathrm{O}_{75} \mathrm{~S}_{6}$ & 5733.5 & GIVEQCCASVCSLYQLENYCN & FVNQHLCGSHLVEALYLVCGERGFFTPKA \\
Insulin Lispro (3) & $\mathrm{C}_{257} \mathrm{H}_{387} \mathrm{~N}_{65} \mathrm{O}_{76} \mathrm{~S}_{6}$ & 5808 & GIVEQCCTSICSLYQLENYCN & FVNQHLCGSHLVEALYLVCGERGFFTKPT \\
Insulin glulisine (4) $\mathrm{C}_{258} \mathrm{H}_{384} \mathrm{~N}_{64} \mathrm{O}_{78} \mathrm{~S}_{6}$ & 5823 & GIVEQCCTSICSLYQLENYCN & FVKQHLCGSHLVEALYLVCGEGFFYTPET \\
Insulin aspart (5) & $\mathrm{C}_{256} \mathrm{H}_{381} \mathrm{~N}_{65} \mathrm{O}_{79} \mathrm{~S}_{6}$ & 5825.8 & GIVEQCCTSICSLYQLENYCN & FVNQHLCGSHLVEALYLVCGERGFFYTDKT \\
Insulin glargine (6) & $\mathrm{C}_{267} \mathrm{H}_{404} \mathrm{~N}_{72} \mathrm{O}_{78} \mathrm{~S}_{6}$ & 6063 & GIVEQCCTSICSLYQLENYCG & FVNQHLCGSHLVEALYLVCGERGFFYTPKTRR \\
\hline
\end{tabular}

Table 2. Peak capacity achieved in RPLC, IEX and HILIC for the different trastuzumab samples

\begin{tabular}{llll}
\hline Peak capacity & RPLC & IEX & HILIC \\
\hline Intact & 55 & 79 & 18 \\
Reduced & 73 & 64 & 82 \\
IdeS digested & 65 & 74 & 85 \\
IdeS digested + reduced & 62 & - & 106 \\
\hline
\end{tabular}

